\title{
BIG GAME HUNTING IN THE ANDROMEDA GALAXY: IDENTIFIYING AND WEIGHING BLACK HOLES IN LOW MASS X-RAY BINARIES
}

\begin{abstract}
R. Barnard ${ }^{1}$
RESUMEN

Hemos desarrollado una nueva técnica para identificar masas de agujeros negros en binarias de rayos $\mathrm{X}$ de baja masa y lo aplicamos a observaciones XMM-Newton de dos fuentes de rayos X en M31. En particular buscamos espectros de potencia de baja tasa de acreción que son muy similares para todas LXMB, siendo la primaria un agujero negro o una estrella de neutrones. Las estrellas neutrónicas galácticas, LXMB, presentan estos PDS distintivos a muy bajas luminosidades $\left(\sim 10^{36} \mathrm{erg} \mathrm{s}^{-1}\right)$; mientras que los LMXB de agujeros negros los pueden presentar a luminosidades $>10^{38} \mathrm{erg} \mathrm{s}^{-1}$. Siguiendo el trabajo de van der Klis (1994) asumimos la máxima tasa de acreción (como una fracción del límite de Eddington) para los PDS de baja tasa de acreción que es constante para todas las LXMB, obteniendo un valor empírico de $\sim 10 \%$ de Eddington. Hasta ahora descubrimos dos candidatos a binarias de agujero negro en M31 que presentan PDS de baja tasa de acreción de hasta $3 \times 10^{38}$ y $5 \times 10^{37} \mathrm{erg} \mathrm{s}^{-1}$. Si asumimos que el límite es $<10 \%$ de Eddington entonces tendrán masas mínimas de 20 y $4 \mathrm{M}_{\odot}$, respectivamente. Además cualquier LXMB que presenta PDS de baja tasa de acreción con una luminosidad $>5 \times 10^{37} \mathrm{erg} \mathrm{s}^{-1}$ probablemente tendrá un agujero negro como su primaria.
\end{abstract}

\section{ABSTRACT}

We have devised a new technique for identifying stellar mass black holes in low mass X-ray binaries, and have applied it to XMM-Newton observations of two X-ray sources in M31. Iri particular we search for low accretion rate power density spectra; these are very similar for all LMXB, whether the primary is a black hole or a neutron star. Galactic neutron star LMXB exhibit these distinctive PDS at very low luminosities $\left(\sim 10^{36}\right.$ erg $\mathrm{s}^{-1}$ ) while black hole LMXB can exhibit them at luminosities $>10^{38} \mathrm{erg} \mathrm{s}^{-1}$ ! Following the work of van der Klis (1994), we assume a maximum accretion rate (as a fraction of the Eddington limit) for low accretion rate PDS that is constant for all LMXB, and obtain an empirical value of $\sim 10 \%$ Eddington. We have so far discovered two candidate black hole binaries in M31, exhibiting low accretion rate PDS at up to $3 \times 10^{38}$ and $5 \times 10^{37} \mathrm{erg} \mathrm{s}^{-1}$. If we assume that they are at $<10 \%$ Eddington, they have minimum masses of 20 and $4 \mathrm{M}$. respectively. Furthermore, any LMXB exhibiting a low accretion rate power density spectrum at a luminosity $>5 \times 10^{37} \mathrm{erg} \mathrm{s}^{-1}$ is likely to have a black hole primary.

Key Words: BLACK HOLE PHYSICS - GALAXIES: INDIVIDUAL (M31) - X-RAYS: BINARIES

\section{INTRODUCTION}

It has long been known that the power density spectra of low luminosity low mass ray binaries (LMXB) with neutron star primaries are strikingly similar to the PDS of black hole LMXB in their low states (van der Klis, 1994). They are characterised by a strong broken power law that is flat at low frequencies; the break frequency ranges over 0.01-0.1 $\mathrm{Hz}$ (van der Klis 1994). At higher accretion rates, the sub-Hertz PDS of black hole LMXB and neutron star LMXB are characterised by weak, power law noise. Van der Klis (1994) proposed that the transition between low-state and high state PDS occurs when the accretion rate exceeds a critical fraction of the Eddington accretion rate $\left(\dot{M}_{\text {Edd }}\right)$, that is constant for all LMXB; he suggests $1 \%$, acknowledging

\footnotetext{
${ }^{1}$ The Open University, UK.
}

that this is an order of magnitude estimate.

We have devised a new technique for identifying stellar-mass black holes in LMXB following this work. We define $f$ as the accretion rate as a fraction of the Eddington limit, i.e. $f=\frac{\dot{M}}{\dot{M}_{\mathrm{Edd}}}$, and a critical accretion rate $f_{\mathrm{c}}$ so that LMXB accreting at $f<f_{\mathrm{c}}$ exhibit low accretion rate PDS and high accretion rate PDS are exhibited at $f>f_{c}$. In these preliminary stages of the work, we assume that $f_{c}$ is constant for all LMXB, allowing black hole LMXB to exhibit low accretion rate PDS at considerably higher luminosities than neutron star LMIXB as obserred. In this work we used results and the literature to estimate $f_{c}$ and applied it to two X-ray source's in M31.

The first step was to examine the PDS of X-ray sources in one of our XMM-Newton observations of 


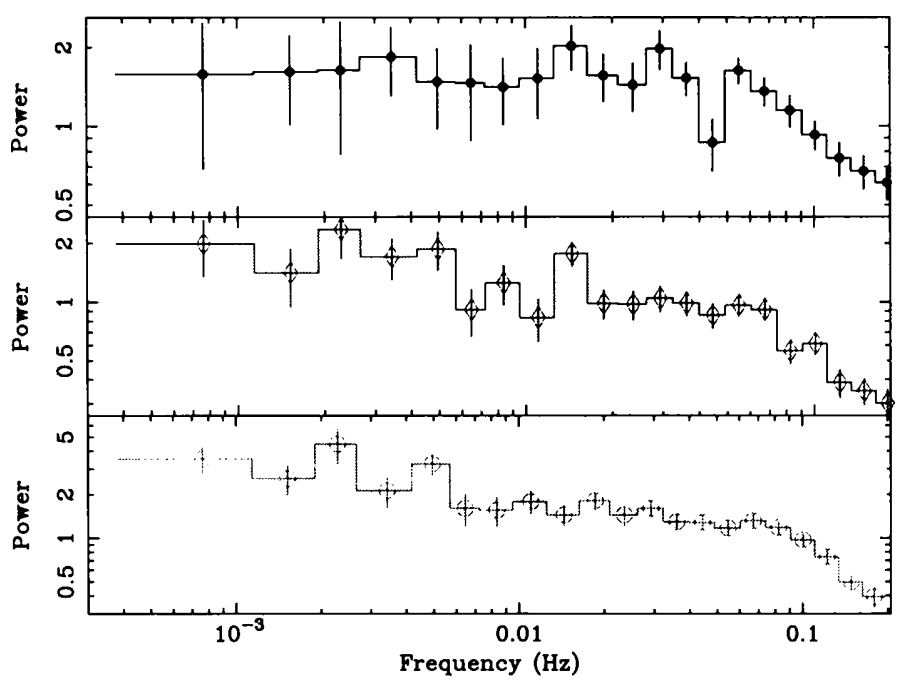

Fig. 1. Power density spectra of the two M31 black hole candidates in M31 in from observations 1, 3 and 4. The left panel shows the PDS of BH1 in observations 1 (top), 3 (middle) and 4 (bottom) with an expected noise level of 0 . All three PDS are characteristic of a low accretion rate, even though the luminosity ranges from $1-310^{38}$ $\operatorname{erg} \mathrm{s}^{-1}$

the core of M31 that were associated with globular clusters; these are likely to be LMXB with neutron star primaries, and the masses of these neutron stars are likely to be around $1.4 \mathrm{M}_{\odot}$. There were seven such sources, with luminosities ranging from $\sim 210^{37}$ erg s${ }^{-1}$ to $\sim 110^{38} \mathrm{erg} \mathrm{s}^{-1}$ in Observation 4 (Barnard et al., 2003). None of these exhibited the broken PDS characteristic of low accretion rates, suggesting that $f_{\mathrm{c}}<\sim 0.1$.

A vital clue was obtained from reviewing the published analysis of the Galactic neutron star LMXB 4U 1705-44. It exhibited a low state PDS in the faintest of four EXOSAT observations, but not in the next faintest; the respective $1-20 \mathrm{keV}$ fluxes were $1.310^{-9}$ and $1.810^{-9} \mathrm{crg} \mathrm{cm}^{-2} \mathrm{~s}^{-1}$ (Langmeier et al., 1987, 1989). Hence, an accurate distance would yield a tight constraint on $f_{c}$. Using a distance to 4U 1705-44 of $8.9 \pm 30 \%$ (Cornelisse et al., 2003), $f_{\mathrm{c}}=0.08_{-0.05}^{+0.08}$, assuming a $1.4 \mathrm{M}$ : primary. Finally V404 Cygni, a Galactic black hole LMXB, exhibited a low state PDS during its outburst at $\sim 10^{38} \mathrm{erg}$ $\mathrm{s}^{-1}$; it has a most probable mass of $12 \mathrm{M}_{\odot}$, implying that $f_{c}>0.05$ (Oosterbroek ct al., 1997, and references within). Our results are consistent with a constant $f_{\mathrm{c}}$ of 0.1 , however it is possible that black hole and neutron star LMXB have different vales of $f_{c}$.
The first X-ray source to be tested, RX J0042.3+4115 (BH1), was straightforward in that a low-rate PDS was observed at luminosities of $1-310^{38} \mathrm{erg} \mathrm{s}^{-1}$, i.e $f \geq \sim 1$ for a $1.4 \mathrm{M}_{\odot}$ neutron star (Barnard et al., 2003)! If we assume that $f_{\mathrm{c}}=$ 0.1 , then the mass of the primary is $>20 \mathrm{M}_{0}$

Identification of the second black hole system was complicated by the fact that the point source in the XMM-Newton observations is resolved by Chandra into two sources. The northern source is known to be transient, while the southern source is associated with a globular cluster (Kong et al., 2002). Hence the northern source is most likely to contain a black hole. XMM-Newton power spectra showed a high state PDS at $310^{37} \mathrm{erg} \mathrm{s}^{-1}$ in the first observation, and yet a low state PDS at $1.210^{38} \mathrm{erg} \mathrm{s}^{-1}$ in the fourth observation! Since we know that two sources are involved, the only way we can understand it is that the component exhibiting the low-state PDS is absent in Observation 1 but present in Observation 4. We made lightcurves of the two sources using 35 Chandra observations; 11 of these were near the $4 \mathrm{XMM}$ observations. We found that the northern source is absent in Observation 1 , while in Observation 4 it contributed $5.310^{37} \mathrm{erg} \mathrm{s}^{-1}$, and the lowstate component to the PDS (Barnard, Kolb \& Osborne, 2004). Assuming that the northern source (BH2) was at less than $10 \% \mathrm{~L}_{\mathrm{Edd}}$, the mass of the primary is $>4 \mathrm{M}_{\odot}$. The theoretical upper limit to the mass of a neutron star is $3.1 \mathrm{M}_{\odot}$, hence the primary of the northern source is a likely black hole candidate.

We have developed a new technique for identifying extra-galactic black holes in LMXB, and obtaining crude minimum masses for them. This technique is potentially very sensitive; we have observed low state PDS for $\mathrm{X}$-ray sources with fluxes down to a few $10^{-14} \mathrm{erg} \mathrm{cm}^{-2} \mathrm{~s}^{-1}$ with XMM. However, we need to survey a large sample of LMXB with known masses to test our assumptions.

\section{REFERENCES}

Barnard, R., Osborne J. P., Kolb, U., Borozdin, K., A\&A, 2003, 405, 505

Barnard, R., Kolb, U., Osborne, J. P., 2004. A\&A, submitted

Cornelisse, R., in't Zand, J. J. M., Verbunt, F., et al., 2003, A\&A, 405, 1033

Kong, A. H. K., Garcia, M. R., Primini, F. A., et al., 2002, ApJ, 577,738

van der Klis, M., 1994, ApJS, 92, 511

Robin Barnard: Department of Physics and Astronomy, The Open University, Walton Hall, Milton Keynes Bucks, MK7 6AA, UK. (r.barnard@open.ac.uk). 